\title{
Mercury release from a Sn-Cu-Zn free amalgam
}

\begin{abstract}
The mercury controversy related to dental amalgam is still continuing. In Malaysia, part of this controversy has been attributed to a recently - introduced dental amalgam claimed to be non-mercury releasing and causing no mercury toxicity. The purpose of this study was to investigate whether this amalgam, Composil, was indeed non-mercury releasing. Six specimens each of Composil and a control (GS-80) were incubated at $37^{\circ} \mathrm{C}$ in deionised-distilled water. The daily mercury release was determined over a four-week study period using the stationary cold-vapour atomic absorption spectrometric method. The mean mercury release of Composil was $30.9 \mu \mathrm{g} / \mathrm{cm}^{2} /$ $24 \mathrm{hr}$ whilst that of GS- 80 was $0.9 \mu \mathrm{g} / \mathrm{cm}^{2} / 24 \mathrm{hr}$ and the difference was found to be highly significant $(\mathrm{P}<0.001)$. Results of this study therefore did not substantiate the manufacturer's claim. The release of mercury from amalgam restorations and their implications in clinical practice were also discussed.
\end{abstract}

\section{Introduction}

Recently, a tin, copper and zinc free amalgam alloy comprising of only silver and mercury was introduced to the dental profession in Malaysia. Amongst the list of claimed superior physical and mechanical properties was the claim that it gave 'no mercury toxicity' as it was a non-mercury releasing material. The manufacturer attributed this to the 'highly reactive silver' which would instantly 'freeze mercury' thereby resulting in an a malgam that did not contain any unreacted free mercury. It was even claimed that burnishing could be carried out without fear of sur facing out any mercury.

A non-mercury releasing amalgam will have significant implications for the dental profession as dental amalgams are still popularly and widely used for the restoration of posterior teeth. More recently, there has again been increased professional and public concern over mercury release from amalgams (1) because of the long recognised toxic potential of

\author{
J.L. Lui ${ }^{*}$ \\ Professor and Head \\ Department of Conservative Dentistry \\ Faculty of Dentistry \\ S.L. Tong \\ Department of Chemistry \\ Faculty of Science \\ S.K. Teh \\ Department of Mechanical Engineering \\ Faculty of Engineering \\ University of Malaya \\ 59100 Kuala Lumpur \\ Malaysia \\ *Corresponding author
}

Key-words: dental amalgam, amalgam restoration, mercury release, mercury toxicity.

mercury. However, the biological significance of release of mercury from amalgam restorations is unknown except for some local hypersensitivity and allergic reactions (2). Whatever the toxicity and toxic potential, it would therefore be desirable for the amalgam restoration to possess low mercury release and it is certainly a great advantage if the amalgam is indeed non-mercury releasing.

The purpose of this study was to investigate whether this new amalgam material was non-mercury releasing by in vitro determination of the level of any released mercury into deionised-distilled water.

\section{Materials and Methods}

The test amalgam material was marketed as Composil (Dencare Products M'sia Sdn. Bhd., Petaling Jaya, Malaysia). Another commercially available amalgam material, GS-80 (Southern Dental Industries Ltd., Bayswater, Australia), was used as the control. Composil, as stated in 
the manufacturer's literature, was a blended composition of a highly reactive silver of very fine micron sized particles. The GS-80 was an admix alloy of a high copper non gamma 2 spherical alloy and a medium copper fine grain lathe-cut alloy containing $40 \%$ silver, $31.2 \%$ tin, $28.6 \%$ copper and $0.2 \%$ zinc.

The amalgam specimens were prepared from capsules according to the manufacturers' instructions by mechanically triturating in a Silamat amalgamator (Vivadent, Schaan/F1, Liechtenstein). Composil was triturated for 5 secs. whilst the mixing time for GS-80 was 8 secs. The mixed amalgam was packed into a highly-polished stainless steel split die firmly placed on a clean glass slab. The die had internal dimensions measuring $8 \mathrm{~mm}$ in length and $4 \mathrm{~mm}$ in diameter producing a calculated surface area of $1.25 \mathrm{~cm}^{2}$ in each cylindrical specimen. To ensure standardisation across specimens, condensation was carried out using the Piezon Master 404 (Electro Medical Systems SA, Le Sentier, Switzerland) rather than manually. The die was packed to excess which was then removed with a sharp scalpel. Six specimens were prepared from each amalgam material.

The amalgam specimens were allowed to set for the recommended time and then washed with $10 \mathrm{~mL}$ of deionised-distilled (dd.) water with moderate agitation for 1 minute. Each specimen was then transferred to a capped 15-mL Teflon vial (Cole Palmer, USA) followed by addition of $10 \mathrm{~mL}$ of dd. water. The specimens in the vials were incubated in an incubator oven at $37^{\circ} \mathrm{C}$ for 24 hours. At the end of the incubation, the specimen was removed and $1 \mathrm{~mL}$ of $0.15 \% \mathrm{w} / \mathrm{v}$ potassium dichromate $\left(\mathrm{K}_{2} \mathrm{C}_{2} \mathrm{O}_{7}\right)$ in $8 \mathrm{M} \mathrm{HNO}_{3}$ was added in each vial to stabilize the mercury. The solution was transferred to a clean glass vial and normally analysed on the same day. The Teflon vials were then cleaned with dichromate-nitric acid and distilled water before being reused for the next incubation. Six determinations of 24-hour mercury release were done for each specimen per week over a four-week period.

A stationary cold-vapour atomic absorption method (3) using a set of four pre-calibrated $4-\mathrm{cm}$ rectangular silica cells (Spectrasil, Thermal Syndicate Limited, England) was employed for the mercury determination. The method is based on the principle of equilibrium partitioning of mercury reduced by the use of stannous chloride between the aqueous and gas phases in the stoppered silica cell. Direct atomic absorption measurement using an atomic absorption spectrophotometer (Model-2380, Perkin-Elmer, U.S.A.) was taken by allowing the mercury resonant light beam to pass through the vapour phase of the system while non-atomic absorption was corrected using an automatic background corrector. A mercury hollow cathode with a lamp current of $3 \mathrm{~mA}$, band width of $0.75 \mathrm{~nm}$ and wavelength of $253.7 \mathrm{~nm}$ was used.
Analytical reagent grade chemicals and dd. water were used for the preparation of solutions. The stock mercury solution $(1000 \mu \mathrm{g} / \mathrm{L})$ was prepared by dissolving $1.354 \mathrm{~g}$ mercury (II) chloride in $50 \mathrm{~mL}$ of concentrated hydrochloric acid and then diluting to $1 \mathrm{~L}$. The working standard of $0.500 \mathrm{ug} / \mathrm{mL}$ was prepared weekly by appropriate dilution from this solution with $0.8 \mathrm{M} \mathrm{HNO}_{3}-0.015 \%$ $\mathrm{K}_{2} \mathrm{Cr}_{2} \mathrm{O}_{7}$ so lution. The reductant consisted of $10 \%$ (w/v) $\mathrm{SnCl}_{2}$, and $5 \%$ (w/v) $\mathrm{NaC} 1$ in $2 \mathrm{M} \mathrm{H}_{2} \mathrm{SO}_{4}$.

To obtain the calibration graphs, $2.50 \mathrm{~mL}$ of $4 \mathrm{M} \mathrm{H}_{2} \mathrm{SO}_{4}$ were pipetted into the cell followed by addition of appropriate volumes (20 to $200 \mu \mathrm{L}$ ) of the working standard $\mathrm{Hg}$ (II) corresponding to the amounts of $10 \mathrm{ng}$ to $100 \mathrm{ng} \mathrm{Hg}$ in the cells. Dd. water was added to make up a total volume of $5.00 \mathrm{~mL}$. This was followed by the addition of $0.30 \mathrm{~mL}$ of $10 \%$ hydroxylamine sulphate $\left[\left(\mathrm{NH}_{2} \mathrm{OH}\right)_{2} \cdot \mathrm{H}_{2} \mathrm{SO}_{4}\right]$, mixed briefly to destroy the oxidant present and finally $0.30 \mathrm{~mL}$ of the $\mathrm{SnC}_{2}$ reductant was introduced. The cell was tightly stoppered and all four cells were shaken together at uniform speed for $2 \mathrm{~min}$. The cell was placed in the holder fixed to the atomic absorption spectrophotometer and steady state readings were obtained after $2 \mathrm{~min}$ standing. Determinations of mercury release in the incubated samples followed the same procedures as described above using $20 \mu \mathrm{L}$ of the Composil and $1.00 \mathrm{~mL}$ of the GS-80 sample solutions. These volumes were predetermined so as to obtain the optimum mercury atomic absorption signal in the sample cells.

In order to check for possible mercury contamination in the experimental process, two blank controls containing only $10 \mathrm{~mL}$ dd. water were subjected to identical treatment starting from the initial incubation stage.

\section{Results}

The weekly and overall mean mercury release of Composil and GS-80, expressed as $\mu \mathrm{g} / \mathrm{cm}^{2} / 24 \mathrm{hr}$, is presented in the Table. The daily average mercury release of the six Composil and six GS-80 specimens was first determined. From these, the weekly and overall mean of both amalgams were derived. The inter-weekly differences for Composil and GS-80 respectively were found to be not significant at the $95 \%$ confidence level by use of the Student's t test. However, the difference in the amount of mercury released by Composil $\left(30.90 \mu \mathrm{g} / \mathrm{cm}^{2} / 24 \mathrm{hr}\right)$ and GS-80 $\left(0.90 \mu \mathrm{g} / \mathrm{cm}^{2} / 24 \mathrm{hr}\right)$ during the entire 4 -week period was found to be highly significant $(\mathrm{p}<0.001)$.

\section{Discussion}

The stationary cold-vapour method for determination of mercury is basically simpler in operation than many other techniques for rapid mercury determination although simultaneous background correction is essential. The tech- 
TABLE

Weekly and overall mean mercury release over a four-week study period

(standard deviation in parentheses)

\begin{tabular}{|c|c|c|c|}
\hline \multirow{3}{*}{$\begin{array}{l}\text { Period } \\
\text { Week } 1\end{array}$} & \multicolumn{3}{|c|}{$\begin{array}{c}\text { Mean Mercury Release } \\
\mu \mathrm{g} / \mathrm{cm}^{2} / 24 \mathrm{hr}\end{array}$} \\
\hline & $\underset{n=6}{\text { Composil }}$ & \multicolumn{2}{|c|}{$\begin{array}{c}\text { GS }-80 \\
n=6\end{array}$} \\
\hline & $29.16(14.97)$ & 1.89 & $(1.40)$ \\
\hline Week 2 & $34.68 \quad(2.69)$ & 0.57 & $(0.18)$ \\
\hline Week 3 & $32.95 \quad(5.13)$ & 0.50 & $(0.23)$ \\
\hline Week 4 & $27.16 \quad(3.38)$ & 0.58 & $(0.25)$ \\
\hline Overall & $30.90 \quad(9.04)$ & 0.90 & $(0.97)$ \\
\hline
\end{tabular}

nique, possessing a sensitivity of $0.0253 \mathrm{ppb}^{-1}$ and a detection limit of $0.02 \mathrm{ppb}$ or $0.1 \mathrm{ng}$, compares favourably even with the best detection limits for other reported transient cold-vapour absorption methods(3). The use of Teflon vials and the addition of $\mathrm{HNO}_{3}$ and $\mathrm{K}_{2} \mathrm{Cr}_{2} \mathrm{O}_{7}$ ensure very good recovery of released mercury for determination.

The mercury determination was carried out in an environment free from mercury contamination. In order to check any possible contamination, two blank controls containing only $10 \mathrm{~mL}$ dd. water but without any amalgam specimens were included in the investigation. No significant contamination was detected in these two controls during the entire four-week study period.

The test amalgam material, Composil, was claimed to have no mercury toxicity because a solid-solution was supposedly formed between the highly reactive silver and mercury resulting in a hardened Composil restoration that contained no unreacted free mercury. However, the results of this investigation showed that the mean overall mercury release from Composil at $30.9 \mu \mathrm{g} / \mathrm{cm}^{2} / 24 \mathrm{hr}$ was more than 30 times higher than the control GS-80 material with a release of $0.9 \mathrm{ug} / \mathrm{cm}^{2} / 24 \mathrm{hr}(\mathrm{P}<0.001)$. Therefore, this study did not substantiate the manufacturer's claim that Composil was non-mercury releasing. In fact, it confirmed the release of high amounts of mercury as reported in other recent studies $(4,5)$.

The daily intake of mercury in its various chemical forms from food and drink is assumed to be around 20 $\mu \mathrm{g}(6)$. According to the results of this in vitro investigation, the estimated daily release of mercury from a multi- surface Composil restoration, at least during the study period, would potentially be equivalent to or even exceed the daily food and drink intake amount. However, it is possible that the result may be different in a complex in vivo situation. Nonetheless, it is imperative from the results to exercise due care when using biomaterials for the safety of patients.

The release of mercury from dental amalgam is reported to be highest during clinical procedures when liquid mercury is still present; viz, during trituration, condensation and immediate finishing of amalgam restorations (7). Although mercury is consumed when the amalgam solidifies with formation of new phases, it does not prevent the release of mercury even though the amalgam restoration is deemed to be set. Consequently, various workers such as Savre (8) and Abraham et al (9) reported significant increase in mercury vapour in persons with amalgam restorations than those without, a significant increase in mercury after chewing in mouths with amalgams and a significant increase in blood mercury concentration in persons with dental amalgams. These workers also found that the blood mercury concentration was positively correlated to the number as well as surface area of the amalgam restorations. In normal circumstances, it is most unlikely for mercury released from amalgam restorations in dental patients to cause acute poisoning; the signs of which include coughing, breathlessness, chest pain, cyanosis and tremor (10). It is generally agreed, however, that the biological significance on patients of mercury release from amalgam restorations is unknown except for some allergic and 
local hypersensitivity reactions $(2,11-14)$. Various claims of other general symptoms of toxicity such as weakness of concentration, dizziness, nausea, tiredness and headaches may have origins other than mercury toxicity(15).

Currently, amalgam restorations which totally do not release mercury are non-existent. Fortunately, the majority of commercially available dental amalgams release only minute quantities of mercury. Except for the rare individual, there is little evidence to justify the claim that this minute amount of mercury released from amalgam restorations has an adverse or toxic effect on the majority of dental patients. Indeed, the side-effects related to dental amalgams are found to be so infrequent that one case per million has been presumed(16). However, it is important to be aware of the potential effects as mercury has been shown not only to accumulate in dental tissues $(17,18)$ but also in various organs of the body $(19,20)$. The mere presence of mercury in a tissue does not actually imply that a toxic effect will occur as both the dose and local concentration have to be considered(21). It is only in those hypersensitive patients that the immunotoxic effect is possibly not dose-related with the consequential manifestation of an allergic or hypersensitivity reaction.

In general therefore, and unless proven otherwise, dental amalgam is still a safe and reliable filling material in the majority of patients. However, it is important when intending to use amalgam materials to select one, which amongst other desirable properties, is low mercury releasing. And coupled with judicious and competent management of mercury, it is possible to further reduce the frequency of mercury toxicity in clinical practice. Considering the high release of mercury in comparison to another commercially available amalgam material, as shown in this investigation, it is prudent therefore to avoid the use of Composil clinically.

\section{References}

1. Okabe T. Mercury in the structure of dental amalgam. Dent Mater 1987; 3: 1-8.

2. Bauer JG, First HA. The toxicity of mercury in dental amalgam. Calif Dent Assoc J 1982; 10: 47-61.

3. Tong SL. Stationary cold-vapour atomic absorption spectrometric method for mercury determination. Anal Chem 1978; 50: 412-14.
4. Lin J-HC, Mante F, Greener EH. Properties of a Sn and Cu free dental amalgam. Trans Acad Dent Mater 1988; 1: 2829

5. Chew CL, Soh G, Lee AS, Yeoh TS. Comparison of release of mercury from three dental amalgams. Dent Mater 1989; 5: $244-46$.

6. Hamilton EI, Minski MJ. Abundance of the chemical elements in man's diet and possible relations with environmental factors. Sci Total Environ 1972/73; 1: 375-94

7. Frykholm KO. Mercury from dental amalgam: its toxic and allergenic effects and some comments on occupational hygiene. Acta Odontol Scand (Suppl.22) 1957; 15: 36-37.

8. Svare CW. Dental amalgam related mercury vapour exposure. Calif Dental Assoc J 1984; 12: 54-60.

9. Abraham JE, Svare CW, Frank C W. The effect of dental amalgam restorations on blood mercury level. J Dent Res 1984; 63: 71-73

10. Skerfving S, Vostal J. Symptoms and signs of intoxication In: Friberg L, Vostal J, eds. Mercury in the environment. Cleveland: CRC Press, 1972; 93-108.

11. Finne K, Goransson K, Winckler L. Oral lichen planus and contact allergy to mercury. Int J Oral Surg 1982; 11: 23639.

12. Mobacken H, Hersle K, Sloberg K, Thilander H. Oral lichen planus hypersensitivity reactions to dental restoration material. Contact Dermatitis 1984; 10:11-15

13. Burrows D. Hypersensitivity to mercury, nickel and chromium in relation to dental materials. Int Dent J 1986; 30: 30-34.

14. Jolly M, Moule AJ, Bryant RW, Freeman S. Amalgamrelated chronic ulceration of oral mucosa. Br Dent J 1986; 160:434-37.

15. Trakhtenberg IM. Chronic effects of mercury on organisms Washington, DC: U.S. Department of Health, Education and Welfare. DHEW Publication No.(NIH) 74, 1974; 399.

16. Donovan TE, Handlers JP. Arational policy on the use of dental amalgam. Calif Dent Assoc J 1984; 12: 10-13.

17. Massler M, Barber TK. Action of amalgam on dentin. J Am Dent Assoc 1953; 47: 415-22.

18. Soremark R, Wing K, Olson K, Goldin J. Penetration of metallic ions from restorations into teeth. J Prosthet Dent 1968; 20: 531-40.

19. Eggleston D, Nylander M. Correlation of dental amalgam with mercury in brain tissue. J Prosthet Dent 1987; 58: 7047.

20. Nylander M, Friberg L, Eggleston D, Bjorkman L. Mercury accumulation in tissues from dental staff and controls in relation to exposure. Swed Dent J 1989; 13: 235-43.

21. Bergman M. Side-effects of amalgam and its alternatives: local, systemic and environmental. Int Dent J 1990: 40: 410. 